\title{
Preparation of palladium nanoparticle catalyst in ionic liquid and its catalytic properties for Heck-Mizoroki reaction
}

\author{
Furong Wang, Sisi Tang, Yinghao Yu, Lefu Wang, Biaolin Yin, Xuehui Li* \\ School of Chemistry and Chemical Engineering, State Key Laboratory of Pulp and Paper Engineering of China, South China University of Technology, \\ Guangzhou 510640, Guangdong, China
}

A R T I C L E I N F O

Article history:

Received 4 July 2014

Accepted 3 September 2014

Published 20 December 2014

\section{Keywords:}

Palladium nanoparticles

Heck-Mizoroki reaction

1-Buthyl-3-methylimidazolium lactate

Green ionic liquid

\begin{abstract}
A B S T R A C T
A palladium nanoparticle (PdNPs) catalyst was synthesized in halogen-free anion ionic liquid (1-buthyl-3-methylimidazolium lactate, [Bmim]Lac) by a simple chemical approach. The transmission electron microscopy analysis confirmed the presence of highly dispersed PdNPs in [Bmim] Lac with small average particle size distribution from 2.2 to $3.1 \mathrm{~nm}$. The size of the PdNPs increases with decreasing molar ratio of $[\mathrm{Bmim}] \mathrm{Lac}$ to $\mathrm{Pd}(\mathrm{OAc})_{2}$ and increasing temperature. The activity of PdNPs@[Bmim]Lac catalyst was tested by the Heck-Mizoroki reaction, and the conditions were optimized. The catalyst can efficiently catalyze couplings of various aryl bromides and iodides with different olefins, giving good yields of products under the optimal conditions and be recycled up to six consecutive times.
\end{abstract}

(C) 2014, Dalian Institute of Chemical Physics, Chinese Academy of Sciences. Published by Elsevier B.V. All rights reserved.
The Pd-catalyzed Heck-Mizoroki reaction has been extensively used to prepare functional materials, natural products, and bioactive compounds [1]. Homogeneous Pd catalysts with high activity are traditionally used, but they are difficult to recycle and dispose of on a large scale, have high toxicity, and are expensive [2]. Reusable and sustainable heterogeneous catalysts such as ligand-free Pd catalysts $[3,4]$ have been extensively investigated as an alternative. The use of ionic liquids (ILs) containing imidazolium cations has attracted particular attention [5].

ILs stabilize metal nanoparticles (NPs) and prevent them from becoming inactive [6]. Heterogeneous nanocatalysts stabilized by ILs composed of halogen-free anions are desirable from an environmental perspective [7]. An IL coupled to a lactate anion is reusable, non-toxic, and environmentally benign [8], and we envision that the hydroxyl and carboxyl groups of the lactate stabilize the PdNPs by interacting with the metal surface. Herein, we report the preparation of a PdNP catalyst stabilized by 1-butyl-3-methylimidazolium lactate (PdNPs@[Bmim]Lac) and its use in the Heck-Mizoroki reaction.

The PdNPs@[Bmim]Lac catalyst was prepared by stirring $\mathrm{Pd}(\mathrm{OAc})_{2}(0.05 \mathrm{mmol})$ and $[\mathrm{Bmim}] \mathrm{Lac}(1 \mathrm{mmol})$ in methanol $(10 \mathrm{~mL})$. The color changed from pale yellow to black indicating formation of PdNPs. UV-Vis absorption spectra of the Pd species before and after reduction were measured at $\lambda=300$ and $800 \mathrm{~nm}$, respectively. The characteristic absorption band for $\mathrm{Pd}(\mathrm{II})$ is at $380 \mathrm{~nm}$. Its disappearance indicated that $\mathrm{Pd}(0)$ had formed [9]. The reducing agent is usually methanol, but to establish if the lactate anion participates in this reduction, electrospray ionization mass spectrometry (ESI-MS) was carried out. This was done at the end of the reaction, however, pyruvate anions (formed from oxidation of the lactate) were not detected.

The effect of the molar ratio of [Bmim] Lac to $\mathrm{Pd}(\mathrm{OAc})_{2}$ and

\footnotetext{
*Corresponding author. Tel/Fax: +86-20-87114707; E-mail: cexhli@scut.edu.cn

This work was supported by the National Natural Science Foundation of China $(21336002,21276094)$ and the Specialized Research Fund for the Doctoral Program of Higher Education (20130172110043).

DOI: 10.1016/S1872-2067(14)60224-2 | http://www.sciencedirect.com/science/journal/18722067 | Chin. J. Catal., Vol. 35, No. 12, December 2014
} 

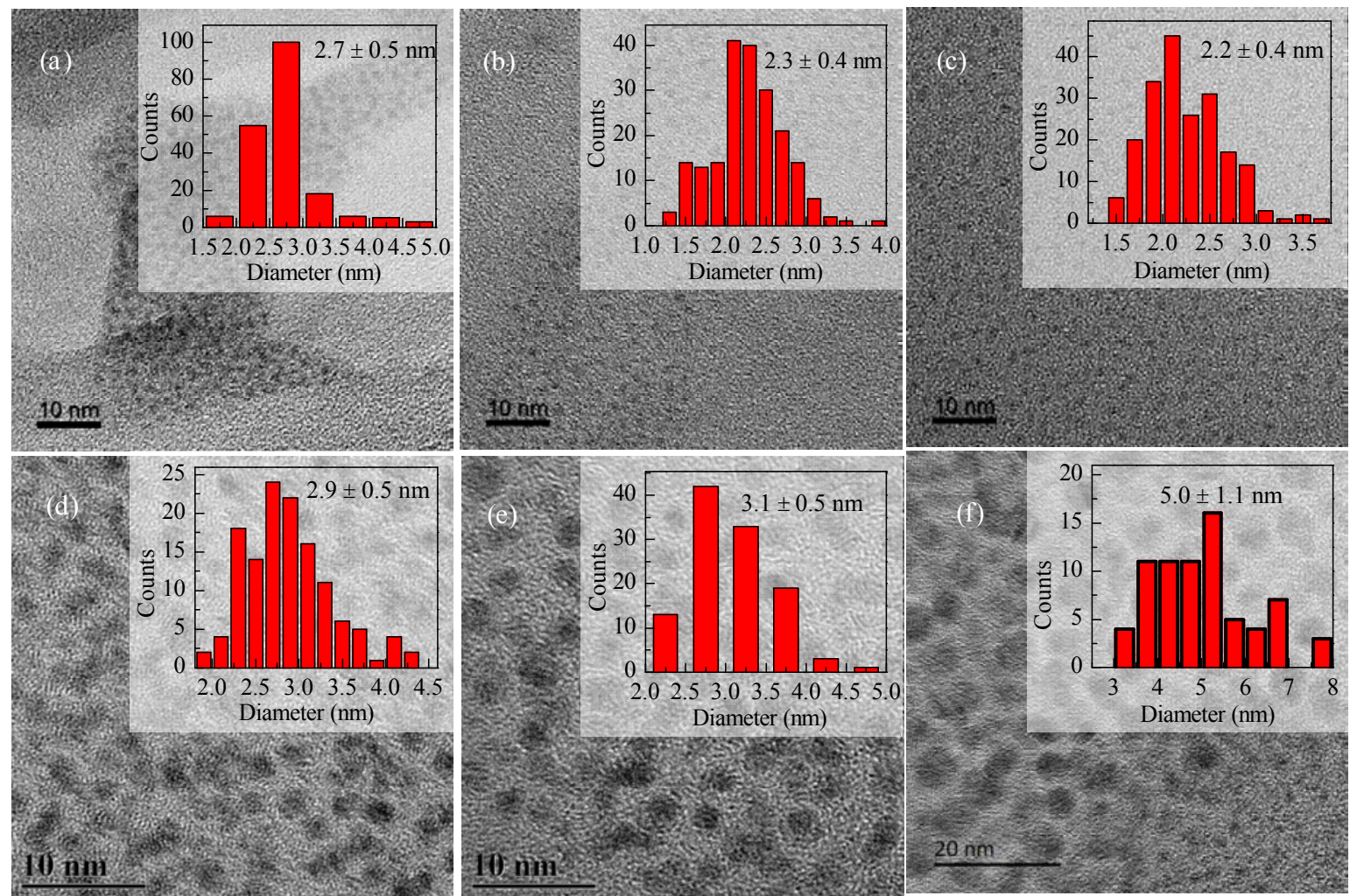

Fig. 1. TEM images of the PdNPs@[Bmim]Lac at different molar ratios of [Bmim]Lac/Pd(OAc)2. (a) 10:1 at r.t.; (b) 20:1 at r.t.; (c) 30:1 at r.t.; (d) 20:1 at $45^{\circ} \mathrm{C}$; (e) $20: 1$ at $65^{\circ} \mathrm{C}$; (f) $20: 1$ at r.t. after six cycles.

the temperature on the size of the PdNPs was examined (Fig. 1 and Table 1). The catalytic activity was assessed using the Heck-Mizoroki reaction with 4-iodoanisole and styrene (Table 1). Transmission electron microscopy (TEM) results showed that when the molar ratio of $[\mathrm{Bmim}] \mathrm{Lac} / \mathrm{Pd}(\mathrm{OAc})_{2}$ was increased from 10:1 to 20:1 the size of the PdNPs decreased from 2.7 to $2.3 \mathrm{~nm}$ (Fig. 1(a) and (b)). The PdNP size decreased from 2.3 to $2.2 \mathrm{~nm}$ as the molar ratio went from 20:1 to 30:1 (Fig. 1 (b) and (c)). We propose that the [Bmim]Lac IL is involved in both dispersing and stabilizing the PdNPs. A layer of ions forms around the Pd atom and provides the necessary electrostatic and steric stabilization. Immediate formation of a surrounding stable layer occurs as the PdNPs grow.

The size of PdNPs increased as the temperature increased

\section{Table 1}

The effect of PdNP size on the Heck-Mizoroki reaction.

\begin{tabular}{lccccc} 
& \\
Entry & $\begin{array}{c}{[\mathrm{Bmim}] \mathrm{Lac} / \mathrm{Pd}(\mathrm{OAc})_{2}} \\
(\mathrm{~mol} / \mathrm{mol})\end{array}$ & $t /{ }^{\circ} \mathrm{C}$ & $\begin{array}{c}\text { Time } \\
(\mathrm{h})\end{array}$ & $\begin{array}{c}\text { Average } \\
\text { size (nm) }\end{array}$ & $\begin{array}{c}\text { Yield }{ }^{\mathrm{C}} \\
(\%)\end{array}$ \\
\hline 1 & $10: 1$ & r.t. & 6 & 2.7 & 80 \\
2 & $20: 1$ & r.t. & 6 & 2.3 & 84 \\
3 & $30: 1$ & r.t. & 6 & 2.2 & 85 \\
4 & $20: 1$ & 45 & 1 & 2.9 & 79 \\
5 & $20: 1$ & 65 & 0.5 & 3.1 & 77
\end{tabular}

Reaction conditions: 4-iodoanisole $(1.0 \mathrm{mmol})$, styrene $(1.5 \mathrm{mmol})$, $\mathrm{Et}_{3} \mathrm{~N}(3.0 \mathrm{mmol}), \mathrm{DMF} / \mathrm{H}_{2} \mathrm{O}(4: 1,6.0 \mathrm{~mL})$ and catalyst $(1 \mathrm{~mol} \% \mathrm{Pd})$ at 90 ${ }^{\circ} \mathrm{C}$ for $9 \mathrm{~h}$. a Determined by GC analysis with hexamethylbenzene as the internal standard.
(Fig. 1(b), (d), and (e)). The higher temperature caused the additional nuclei to form quickly, and their deposition on the

Table 2

Optimization of the Heck-Mizoroki reaction conditions.

\begin{tabular}{|c|c|c|c|c|c|}
\hline Entry & Base & $\begin{array}{c}\text { Solvent } \\
\text { (vol/vol) }\end{array}$ & $\begin{array}{c}{[\mathrm{Pd}]} \\
(\mathrm{mol} \%)\end{array}$ & $t /{ }^{\circ} \mathrm{C}$ & Yield a (\%) \\
\hline 1 & $\mathrm{Et}_{3} \mathrm{~N}$ & toluene & 2.0 & 90 & 12 \\
\hline 2 & $\mathrm{Et}_{3} \mathrm{~N}$ & EtOH & 2.0 & 90 & 27 \\
\hline 3 & $\mathrm{Et}_{3} \mathrm{~N}$ & $\mathrm{i}-\mathrm{PrOH}$ & 2.0 & 90 & 30 \\
\hline 4 & $\mathrm{Et}_{3} \mathrm{~N}$ & DMF & 2.0 & 90 & 39 \\
\hline 5 & $\mathrm{Et}_{3} \mathrm{~N}$ & $\mathrm{DMF} / \mathrm{H}_{2} \mathrm{O}(4: 1)$ & 2.0 & 90 & 92 \\
\hline 6 & $\mathrm{Et}_{3} \mathrm{~N}$ & $\mathrm{H}_{2} \mathrm{O}$ & 2.0 & 90 & 20 \\
\hline 7 & $\mathrm{Na}_{2} \mathrm{CO}_{3}$ & $\mathrm{DMF} / \mathrm{H}_{2} \mathrm{O}(4: 1)$ & 2.0 & 90 & 60 \\
\hline 8 & $\mathrm{NaOH}$ & $\mathrm{DMF} / \mathrm{H}_{2} \mathrm{O}(4: 1)$ & 2.0 & 90 & 62 \\
\hline 9 & $\mathrm{~K}_{2} \mathrm{CO}_{3}$ & $\mathrm{DMF} / \mathrm{H}_{2} \mathrm{O}(4: 1)$ & 2.0 & 90 & 65 \\
\hline 10 & $\mathrm{Na}_{3} \mathrm{PO}_{4} \cdot 12 \mathrm{H}_{2} \mathrm{O}$ & $\mathrm{DMF} / \mathrm{H}_{2} \mathrm{O}(4: 1)$ & 2.0 & 90 & 87 \\
\hline 11 & $\mathrm{Et}_{3} \mathrm{~N}$ & $\mathrm{DMF} / \mathrm{H}_{2} \mathrm{O}(5: 1)$ & 2.0 & 90 & 89 \\
\hline 12 & $\mathrm{Et}_{3} \mathrm{~N}$ & $\mathrm{DMF} / \mathrm{H}_{2} \mathrm{O}(3: 1)$ & 2.0 & 90 & 86 \\
\hline 13 & $\mathrm{Et}_{3} \mathrm{~N}$ & $\mathrm{DMF} / \mathrm{H}_{2} \mathrm{O}(2: 1)$ & 2.0 & 90 & 78 \\
\hline 14 & $\mathrm{Et}_{3} \mathrm{~N}$ & $\mathrm{DMF} / \mathrm{H}_{2} \mathrm{O}(4: 1)$ & 2.0 & 80 & 75 \\
\hline 15 & $\mathrm{Et}_{3} \mathrm{~N}$ & $\mathrm{DMF} / \mathrm{H}_{2} \mathrm{O}(4: 1)$ & 2.0 & 100 & 91 \\
\hline 16 & $\mathrm{Et}_{3} \mathrm{~N}$ & $\mathrm{DMF} / \mathrm{H}_{2} \mathrm{O}(4: 1)$ & 2.0 & 110 & 88 \\
\hline 17 & $\mathrm{Et}_{3} \mathrm{~N}$ & $\mathrm{DMF} / \mathrm{H}_{2} \mathrm{O}(4: 1)$ & - & 90 & 0 \\
\hline 18 & $\mathrm{Et}_{3} \mathrm{~N}$ & $\mathrm{DMF} / \mathrm{H}_{2} \mathrm{O}(4: 1)$ & 0.5 & 90 & 47 \\
\hline 19 & $\mathrm{Et}_{3} \mathrm{~N}$ & $\mathrm{DMF} / \mathrm{H}_{2} \mathrm{O}(4: 1)$ & 1.0 & 90 & 84 \\
\hline 20 & $\mathrm{Et}_{3} \mathrm{~N}$ & $\mathrm{DMF} / \mathrm{H}_{2} \mathrm{O}(4: 1)$ & 1.5 & 90 & 89 \\
\hline 21 & $\mathrm{Et}_{3} \mathrm{~N}$ & $\mathrm{DMF} / \mathrm{H}_{2} \mathrm{O}(4: 1)$ & 3.0 & 90 & 92 \\
\hline
\end{tabular}

Reaction conditions: 4-iodoanisole $(1.0 \mathrm{mmol})$, styrene $(1.5 \mathrm{mmol})$, base $(3.0 \mathrm{mmol})$, solvent $(6.0 \mathrm{~mL})$ for $9 \mathrm{~h}$. aDetermined by GC analysis (hexamethylbenzene as an internal standard). 
existing particles induced the further growth of PdNPs. The size of PdNPs had an effect on the Heck-Mizoroki reaction (Table 1). A higher yield was observed when a smaller PdNP was used. This is because of the larger specific surface area providing more active sites. The preparation conditions of the catalysts were $[\mathrm{Bmim}] \mathrm{Lac} / \mathrm{Pd}(\mathrm{OAc})_{2} \quad(20: 1)$ and r.t. in the following study.

The coupling of 4-iodoanisole with styrene was selected as a model reaction and the conditions were optimized (Table 2). Anhydrous toluene, EtOH, $\mathrm{i}$-PrOH, or DMF $(N, N$-dimethylformamide) gave low yields of less than $40 \%$ at $90{ }^{\circ} \mathrm{C}$ (Table 2 , entries 1-4). When an organic/aqueous co-solvent system $\left(\mathrm{DMF} / \mathrm{H}_{2} \mathrm{O}\right)$ was used the yield increased to $92 \%$ (Table 2, entry 5). This may be because [Bmim]Lac has good dispersion in
$\mathrm{H}_{2} \mathrm{O}$. When $\mathrm{H}_{2} \mathrm{O}$ alone was used as the solvent, a low yield was observed (Table 2, entry 6) possibly because of poor substrate solubility. Different bases were then screened, and $\mathrm{Et}_{3} \mathrm{~N}$ gave the best result (Table 2, entries 7-10). This corresponds to results reported in the literature $[10,11]$. We infer that $\mathrm{Et}_{3} \mathrm{~N}$ has a positive influence on the reaction rate and the catalyst stability as that of tetrabutylammonium bromide (TBAB) reported [12]. In addition, stabilization of the catalytic system with ammonium salts extends the palladacycle life [13]. Different volume ratios of DMF to $\mathrm{H}_{2} \mathrm{O}$ were investigated (Table 2, entries 11-13), and the volume ratio of $4: 1$ is still the best. Similar to literature reported, a temperature of $90{ }^{\circ} \mathrm{C}$ was found to be the optimum (Table 2, entries 14-16) [14]. The coupling reaction does not proceed in the absence of the Pd catalyst (Table 2,

Table 3

Scope of the Heck-Mizoroki reaction.

\begin{tabular}{|c|c|c|c|c|c|}
\hline Entry & $\mathrm{X}$ & $\mathrm{R}_{1}$ & $\mathrm{R}_{2}$ & Time (h) & Yield a (\%) \\
\hline 1 & I & $4-\mathrm{OCH}_{3}$ & & 9 & 92 \\
\hline 2 & I & $4-\mathrm{CH}_{3}$ & & 9 & 93 \\
\hline 3 & I & $4-\mathrm{NO}_{2}$ & & 9 & 82 \\
\hline 4 & I & $\mathrm{H}$ & & 9 & 92 \\
\hline 5 & $\mathrm{Br}$ & 4-CHO & & 9 & 92 \\
\hline 6 & $\mathrm{Br}$ & $4-\mathrm{CN}$ & & 9 & 93 \\
\hline 7 & $\mathrm{Br}$ & $4-\mathrm{OCH}_{3}$ & & 9 & 91 \\
\hline 8 & $\mathrm{Br}$ & $4-\mathrm{NO}_{2}$ & & 9 & 75 \\
\hline 9 & $\mathrm{Br}$ & $2-\mathrm{OCH}_{3}$ & & 11 & 60 \\
\hline 10 & I & $4-\mathrm{OCH}_{3}$ & $\mathrm{COOCH}_{3}$ & 8 & 93 \\
\hline 11 & I & $4-\mathrm{OCH}_{3}$ & $\mathrm{COOC}_{4} \mathrm{H}_{9}$ & 9 & 91 \\
\hline 12 & I & $4-\mathrm{OCH}_{3}$ & $\mathrm{COOH}$ & 7 & 92 \\
\hline 13 & I & $4-\mathrm{CH}_{3}$ & $\mathrm{COOC}_{4} \mathrm{H}_{9}$ & 10 & 87 \\
\hline 14 & I & $4-\mathrm{CH}_{3}$ & $\mathrm{COOH}$ & 7 & 96 \\
\hline $15^{\mathrm{b}}$ & $\mathrm{Cl}$ & $\mathrm{H}$ & & 24 & $<5$ \\
\hline $16^{\mathrm{b}}$ & $\mathrm{Cl}$ & $4-\mathrm{OCH}_{3}$ & & 24 & $<5$ \\
\hline $17^{b}$ & $\mathrm{Cl}$ & $4-\mathrm{NO}_{2}$ & & 24 & $<5$ \\
\hline
\end{tabular}

Reaction conditions: aryl halide $(1.0 \mathrm{mmol})$, olefin $(1.5 \mathrm{mmol}), \mathrm{Et}_{3} \mathrm{~N}(3.0 \mathrm{mmol}), \mathrm{DMF} / \mathrm{H}_{2} \mathrm{O}(4: 1 ; 6.0 \mathrm{~mL})$ and catalyst $(2 \mathrm{~mol} \%$ of Pd $)$ at $90{ }^{\circ} \mathrm{C}$. ${ }^{a}$ Isolated yield for trans product. ${ }^{b}$ Determined by GC analysis with hexamethylbenzene as an internal standard. 
entry 17). The yield increased as the amount of PdNPs increased with a peak at 2.0 mol\% PdNPs (Table 2, entry 5). To summarize, the optimal conditions are $1.0 \mathrm{mmol}$ aryl halide, 1.5 mmol olefin, 2.0 mol\%PdNPs@[Bmim]Lac, 3.0 mmol Et $3 \mathrm{~N}$, and $6.0 \mathrm{~mL} \mathrm{DMF} / \mathrm{H}_{2} \mathrm{O}(4: 1)$ at $90^{\circ} \mathrm{C}$.

The substrate scope of the Heck-Mizoroki reaction was tested with a variety of aryl halides and olefins under the optimized conditions (Table 3). Aryl bromides and iodides substituted with electron-withdrawing $\left(-\mathrm{NO}_{2},-\mathrm{CN},-\mathrm{CHO}\right)$ and electron-donating groups $\left(-\mathrm{OCH}_{3},-\mathrm{CH}_{3}\right)$ are tolerated and give the corresponding products in high yield (Table 3, entries 1-9). Notably, the aryl bromide and iodide with an $-\mathrm{NO}_{2}$ group gave a lower yield than with the $-\mathrm{OCH}_{3}$ group (Table 3 , entry 1 vs entry 3; entry 7 vs entry 8). A similar result has been observed in the literature [15]. The lower yield and longer reaction time observed for the $O$-methoxy analogue may be attributed to steric hindrance (Table 3, entry 9). Again, a similar result has been reported in the literature [16]. Olefins such as acrylic acid and methyl- or butyl-acrylate were very well tolerated. The larger the geometrical size of acrylate ester, requires a longer reaction time $\left(\mathrm{C}_{4} \mathrm{H}_{9}>\mathrm{CH}_{3}>\mathrm{H}\right)$ (Table 3 , entries 10-14). Aryl chlorides give low yields of product with a long reaction time (Table 3, entries 15-17). Thus, the PdNPs@[Bmim]Lac catalyst exhibits good functional group tolerance and high catalytic activity. We infer that [Bmim] Lac and $\mathrm{Et}_{3} \mathrm{~N}$ jointly contribute to the stability and activity of the PdNP catalyst. Formation of PdNPs surrounded by lactate anions provide a coulombic barrier for collisions and stop clusters growing into the metal. In addition, the carboxyl and hydroxyl groups of the lactate may provide sufficient stabilization and disperse the PdNPs.

The reusability of the PdNP catalyst ( $1 \mathrm{~mol} \%$ of Pd) was investigated. After one cycle the products were extracted with diethyl ether and the solvents and $\mathrm{Et}_{3} \mathrm{~N}$ were removed with a rotary evaporator. The catalyst was then used for up to 6 more experiments (Fig. 2). TEM results showed that the average particle size of PdNP increased from 2.3 to $5.0 \mathrm{~nm}$ (Fig. 1(f)). This observation may be attributed to the Ostwald ripening process.

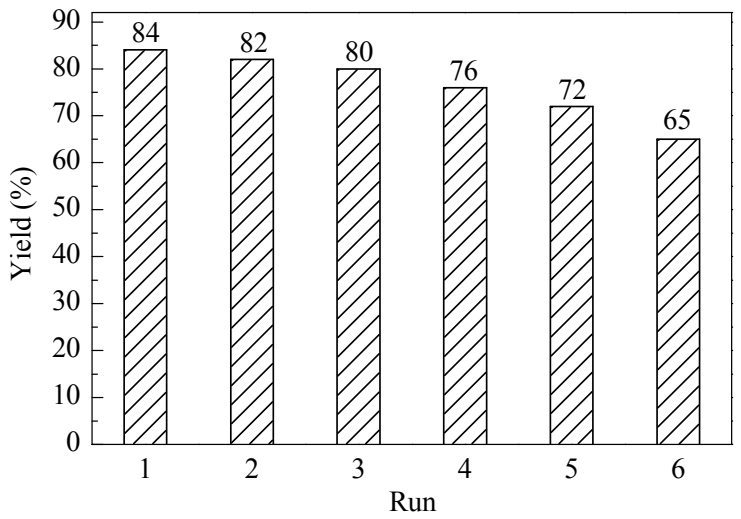

Fig. 2. Recycling tests for the standard reaction. Reaction conditions: 4-iodoanisole $(1.0 \mathrm{mmol})$, styrene $(1.5 \mathrm{mmol})$, catalyst ( $1 \mathrm{~mol} \%$ of Pd), $\mathrm{Et}_{3} \mathrm{~N}(3.0 \mathrm{mmol})$, and $\mathrm{DMF} / \mathrm{H}_{2} \mathrm{O}=4: 1(6.0 \mathrm{~mL})$ at $90{ }^{\circ} \mathrm{C}$ for $9 \mathrm{~h}$.

Atomic absorption spectroscopy (AAS) showed that approximately $0.3 \%$ of the total Pd remains in the ether extract. This $\mathrm{Pd}$ sample was tested in the coupling of 4-iodoanisole and styrene under the optimized conditions and did not exhibit catalytic activity.

In conclusion, we have reported the simple and reproducible preparation of the PdNPs@[Bmim]Lac catalyst using a non-toxic and environmentally benign lactate anion IL as the stabilizer. TEM confirmed the presence of highly dispersed PdNPs in the [Bmim]Lac with an average particle size of 2.2-3.1 nm. This catalyst efficiently catalyzes the Heck-Mizoroki reaction for a range of aryl bromides and iodides with different olefins and can be recycled up to six consecutive times.

\section{References}

[1] Beletskaya I P, Cheprakov A V. Chem Rev, 2000, 100: 3009

[2] Birkholz M N, Freixa Z, Van Leeuwen P W N M. Chem Soc Rev, 2009, 38: 1099

[3] Trzeciak A M, Ziółkowski J J. Coord Chem Rev, 2007, 251: 1281

\section{Graphical Abstract}

Chin. J. Catal., 2014, 35: 1921-1926 doi: 10.1016/S1872-2067(14)60224-2

Preparation of palladium nanoparticle catalyst in ionic liquid and its catalytic properties for Heck-Mizoroki reaction

Furong Wang, Sisi Tang, Yinghao Yu, Lefu Wang, Biaolin Yin, Xuehui Li* South China University of Technology

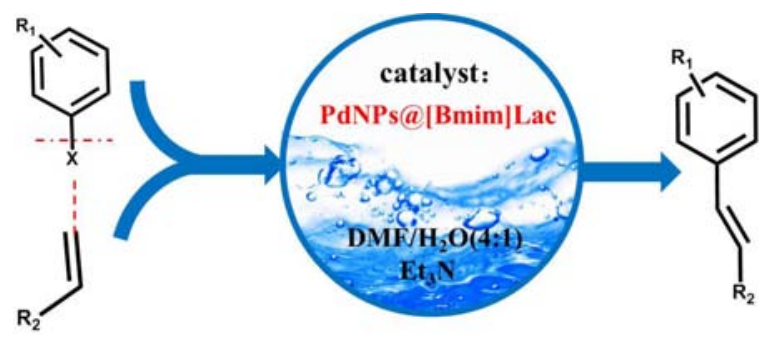

A PdNPs@[Bmim]Lac catalyst was prepared using a non-toxic and environmentally benign lactate anion IL as a stabilizer. The catalyst can be used efficiently for the Heck-Mizoroki reaction of various aryl halides with different olefins and can be reused. 
[4] Liu H F, Jia Z G, Ji S F. Chin J Catal (刘鸿飞, 贾志刚, 季生福. 催化 学报), 2012, 33: 757

[5] Scholten J D, Leal B C, Dupont J. ACS Catal, 2012, 2: 184

[6] Dupont J, Scholten J D. Chem Soc Rev, 2010, 39: 1780

[7] Petkovic M, Seddon K R, Rebelo L P N, Silva Pereira C. Chem Soc Rev, 2011, 40: 1383

[8] Pernak J, Goc I, Mirska I. Green Chem, 2004, 6: 323

[9] Harraz F A, El-Hout S E, Killa H M, Ibrahim I A. J Catal, 2012, 286: 184

[10] Zeng Y, Wang Y H, Xu Y C, Song Y, Jiang J Y, Jin Z L. Catal Lett, 2012, 143: 200
[11] Bakherad M, Keivanloo A, Samangooei S. Chin J Catal (催化学报), 2014, 35: 324

[12] Calo V, Nacci A, Monopoli A, Fornaro A, Sabbatini L, Cioffi N, Ditaranto N. Organometallics, 2004, 23: 5154

[13] Herrmann W A, Brossmer C, Ofele K, Reisinger C P, Priermeier T, Beller M, Fischer H. Angew Chem Int Ed, 1995, 34: 1844

[14] Nie G R, Zhang L, Cui Y C. Chin J Org Chem (聂广瑞, 张否, 崔元臣. 有机化学), 2013, 33: 1734

[15] Luo C C, Zhang Y H, Wang Y G. J Mol Catal A, 2005, 229: 7

[16] Wang J, Mu B, Fu Z H, Wang L, Li T S, Wu Y J. Chin J Catal (王炣, 穆 兵, 付志华, 汪丽, 李铁生, 吴养洁. 催化学报), 2014, 35: 1059

\title{
绿色离子液体中纳米钯催化剂的制备及其催化Heck-Mizoroki反应性能
}

\author{
王芙蓉，唐思思，于英豪，王乐夫，尹标林，李雪辉 ${ }^{*}$ \\ 华南理工大学化学与化工学院, 制浆造纸工程国家重点实验室, 广东广州510640
}

摘要: 发展了在非卤素绿色离子液体1-丁基-3-甲基咪唑离子液体乳酸盐中制备纳米Pd催化剂的简便化学方法. 透射电镜结果表 明, Pd纳米粒子高度分散在 $[\mathrm{Bmim}] \mathrm{Lac}$ 离子液体中, 平均粒径为2.2-3.1 nm. Pd纳米粒子的大小随着体系中 $[\mathrm{Bmim}] \mathrm{Lac}$ 与 $\mathrm{Pd}(\mathrm{OAc})_{2}$ 摩尔比减小和温度升高而增大. 考察了离子液体稳定纳米Pd催化剂(PdNPs@[Bmim]Lac)催化Heck-Mizoroki反应性能, 并对反应 条件进行了优化. 结果表明, 所制备的离子液体稳定的纳米Pd催化剂在优化条件下可高效催化系列卤代芳烃与烯烃的 Heck-Mizoroki反应, 且可循环使用6次.

关键词: 钯纳米粒子; Heck-Mizoroki反应; 1-丁基-3-甲基咪唑离子液体乳酸盐; 绿色离子液体

收稿日期: 2014-07-04. 接受日期: 2014-09-03. 出版日期: 2014-12-20.

*通讯联系人. 电话/传真：(020)87114707; 电子信箱：cexhli@scut.edu.cn

基金来源：国家自然科学基金(21336002和21276094); 高等学校博士学科点专项科研基金(20130172110043).

本文的英文电子版由Elsevier出版社在ScienceDirect上出版(http://www.sciencedirect.com/science/journal/18722067).

钯催化的Heck-Mizoroki反应广泛应用于制备功能 材料、天然产物和生物活性物质的中间体 ${ }^{[1]}$. 该反应经常 采用均相Pd基催化剂,存在催化剂分离回收困难、有毒、 价格昂贵和废弃物处理等问题 ${ }^{[2]}$. 因此, 可持续重复使用 的非均相催化剂如无配位体的Pd催化剂广受关注 ${ }^{[3,4]}$,特 别是在含咪唑阳离子的离子液体领域 ${ }^{[5]}$.

离子液体能够稳定金属纳米粒子, 避免纳米粒子形 成团聚而失活 ${ }^{[6]}$. 从环保角度出发, 开发新型不含卤素阴 离子的离子液体稳定纳米催化剂具有重要意义 ${ }^{[7]}$. 含乳 酸阴离子的离子液体具有可再生、无毒和环境友好等特 性 ${ }^{[8]}$, 我们设想乳酸盐阴离子中含有的羟基和羧基具备 与金属表面相互作用的可能而进一步稳定金属纳米颗 粒. 在此, 我们报道了一种利用1-丁基-3-甲基咪唑乳酸 盐离子液体稳定Pd纳米颗粒 $(\mathrm{PdNPs} @[\mathrm{Bmim}] \mathrm{Lac})$ 的催 化剂的制备方法及其在Heck-Mizoroki反应中的催化性 能.

将Pd(OAc $)_{2}(0.05 \mathrm{mmol}),[\mathrm{Bmim}] \mathrm{Lac}(1 \mathrm{mmol})$ 及甲醇 $(10 \mathrm{~mL})$ 混合并摚拌一定时间. 混合物的颜色由亮黄色变 为黑色表明形成了 Pd纳米粒子. 还原过程中 $\mathrm{Pd}(\mathrm{II})$ 的还 原程度利用紫外-可见吸收光谱 $(\lambda=300-800 \mathrm{~nm})$ 进行检
测. 由于Pd(II)在 $380 \mathrm{~nm}$ 处有特征吸收峰, 该峰消失表明 $\operatorname{Pd}(\mathrm{II})$ 完全还原为 $\operatorname{Pd}(0)^{[9]}$. 甲醇通常用作 $\operatorname{Pd}(\mathrm{II})$ 还原为 $\operatorname{Pd}(0)$ 的还原剂. 为了考察乳酸根阴离子是否参与该还原 过程, 我们用ESI-MS检测还原后混合物, 未发现乳酸根 阴离子被氧化的产物丙酮酸根阴离子. 这说明在制备催 化剂过程中乳酸根阴离子很稳定, 没有参与 $\mathrm{Pd}(\mathrm{II})$ 的还 原.

考察了 $[\mathrm{Bmim}] \mathrm{Lac}$ 与 $\mathrm{Pd}(\mathrm{OAc})_{2}$ 的摩尔比和制备温度 对所形成Pd纳米粒子的影响(图1和表1), 并测试了所制 催化剂对4-碘苯甲醚与苯乙烯Heck-Mizoroki 反应的催 化性能. 由图1可见, Pd纳米粒子的粒径随着 [Bmim]Lac 用量增加而减小, 与纳米颗粒大小随稳定剂用量增加而 减小的规律一致. 当 $[\mathrm{Bmim}] \mathrm{Lac} / \mathrm{Pd}(\mathrm{OAc})_{2}$ 摩尔比从 $10: 1$ 升至 20:1时, Pd纳米粒子的平均粒径从 $2.7 \mathrm{~nm}$ 降至 $2.3 \mathrm{~nm}$ (图1 (a)和(b)); 当该摩尔比继续升至 30:1 时, Pd纳米粒子 的平均粒径从 $2.3 \mathrm{~nm}$ 略降到 $2.2 \mathrm{~nm}$ (图 1 (b)和(c)). 我们认 为, $[\mathrm{Bmim}] \mathrm{Lac}$ 离子液体在反应体系中具有分散和稳定 Pd纳米粒子的作用, 可在Pd纳米粒子周围形成提供位阻 和电阻保护的离子层. 当Pd纳米粒子长大时, 在它周围 迅速形成稳定层. 
另外, Pd纳米粒子的粒径随着温度升高而增大(图1 (b), (d)和(e)). 这是因为Pd纳米粒子在第一次成核后, 较 高温度下,其他核的形成及其与前驱体的共沉积(在已形 成的核上)速率较快, 导致Pd纳米粒子快速生长而长大. 由表 1 可见, $\mathrm{Pd}$ 粒子越小, 产物产率越高, 这是因为在 $\mathrm{Pd}$ 用 量相同时,平均粒径越小的Pd纳米颗粒体系可提供更多 的活性中心. 因此,下文采用粒径较小的催化剂(图1(b)).

以4-碘苯甲醚和苯乙烯的偶联作为模型反应优化 了反应条件(表2). 溶剂和碱是影响反应结果的重要因 素. 在 $90^{\circ} \mathrm{C}$ 下, 当使用无水溶剂如甲苯、乙醇、异丙醇和 $\mathrm{N}, \mathrm{N}$-二甲基甲酰胺(DMF)时,产物产率小于 $40 \%$ (表 2, 实 验 1-4), 当使用有机溶剂和水的混合溶剂时，如 $\mathrm{DMF} / \mathrm{H}_{2} \mathrm{O}$, 目标产物的产率可达 $92 \%$ (表 2 , 实验 5 ). 这可 能是因为 $[\mathrm{Bmim}] \mathrm{Lac}$ 离子液体与水有良好的互溶性, 离 子液体稳定的纳米催化剂体系可高度分散. 然而, 仅仅 用水作为溶剂时产率很低(表 2 , 实验 6 ), 可能是因为反应 底物在水中溶解度很小的缘故. 当对碱进行优化时, 发 现 $\mathrm{Et}_{3} \mathrm{~N}$ 用作碱最佳(表 2, 实验 7-10), 与文献结果一致 ${ }^{[10,11]}$. 此外,有研究表明,四丁基溴化铵(TBAB)对该类偶 联反应的反应速率及催化剂的稳定性有促进作用 ${ }^{[12]}$; Herrmann等 ${ }^{[13]}$ 也发现, 使用铵盐可延长Pd催化剂的使用 寿命. 本文研究结果发现, 以 $\mathrm{Et}_{3} \mathrm{~N}$ 为碱, $\mathrm{DMF}$ 与 $\mathrm{H}_{2} \mathrm{O}$ 体积 比为 $4: 1$, 在 $90{ }^{\circ} \mathrm{C}$ 时, 该催化体系效率最高 (表 2 , 实验 11-16). 温度影响反应的变化规律与文献相同 ${ }^{[14]}$. 我们 还考察了催化剂使用量对反应的影响(表2, 实验17-21). 结果发现, 不加Pd催化剂, 偶联反应则不能进行(表 2 , 实 验 17); 随着催化剂使用量增加, 产物产率逐渐增加, 至2 $\mathrm{mol} \%$ 时最高(表 2 , 实验 5 ). 因此, 最佳反应条件为: 1.0 mmol芳基卤, 1.5 mmol烯烃,2.0mol\%PdNPs@[Bmim]Lac, $3.0 \mathrm{mmol} \mathrm{Et}_{3} \mathrm{~N}$ 和 $6.0 \mathrm{mLDMF} / \mathrm{H}_{2} \mathrm{O}(4: 1)$, 温度为 $90^{\circ} \mathrm{C}$.

基于以上研究结果,我们对PdNPs@[Bmim]Lac催化 芳基卤与烯烃发生Heck-Mizoroki反应的底物范围进行 了拓展(表3). 结果表明, 该催化剂对一系列具有不同官 能团的底物具有普适性. 即含吸电子基 $\left(-\mathrm{NO}_{2},-\mathrm{CN}\right.$,
$-\mathrm{CHO})$ 、电中性基团 $(\mathrm{H})$ 或供电子基团 $\left(-\mathrm{OCH}_{3},-\mathrm{CH}_{3}\right)$ 的 溴苯和碘苯能与苯乙烯高效偶联, 目标产物的产率较高 (表 3 , 实验 1-9). 含供电子基团 $-\mathrm{OCH}_{3}$ 的反应物的偶联效 率较含吸电子基团 $-\mathrm{NO}_{2}$ 的溴苯和碘苯更高(表 3 , 实验 1 和 3,7 和 8$)$, 与文献结果一致 ${ }^{[15]}$. 此外, 我们发现, 邻甲氧 基溴苯的活性较低,这是因为邻位基团空间位阻较强的 缘故(表3, 实验9), 与文献[16]结果相似. 此外, 含供电子 基团 $\left(-\mathrm{OCH}_{3},-\mathrm{CH}_{3}\right)$ 的碘苯能高效地与丙烯酸、丙烯酸甲 基和丙烯酸丁酯偶联. 对丙烯酸酯化合物而言, 随着取 代基团碳数的增加, 获得接近目标化合物产率的反应时 间增加(表3, 实验 10-14). 当以芳基氯化物为反应底物时, 产物产率小于 5\% (表 3, 实验 15-17). 由此可见, PdNPs@[Bmim]Lac催化剂对反应底物的官能团具有较 好的普适性. 我们推断, [Bmim] $\mathrm{Lac}$ 与 $\mathrm{Et}_{3} \mathrm{~N}$ 的协同有助于 提高催化剂的稳定性及活性. 围绕在Pd纳米粒子周围的 乳酸阴离子对纳米粒子碰撞形成库仑屏障, 阻碍Pd金属 簇的进一步团聚. 此外, 通过提供孤对电子, 乳酸阴离子 中的羧基和羟基均能进一步促进Pd纳米粒子的稳定性.

图2考察了4-碘苯甲醚与苯乙烯Heck-Mizoroki反应 中催化剂的重复使用性能,催化剂Pd的使用量为 $1 \mathrm{~mol} \%$. 在反应结束后, 反应产物可以用乙醚完全萃取. 将残留 物旋蒸去除溶剂及Et $\mathrm{t}_{3} \mathrm{~N}$ 后, 所得PdNPs@[Bmim]Lac催化 剂直接用于下一次反应. 由图2可见,催化剂可循环使用 6次. TEM结果显示, 使用6次后, Pd纳米粒子的粒径从原 来的 $2.3 \mathrm{~nm}$ 增至 $5.0 \mathrm{~nm}$ (图1(f)), 这可能是因为奥斯特瓦 尔德熟化过程所致. 原子吸收光谱测定表明在乙醚萃取 液中含有 $0.3 \%$ 的Pd. 旋转蒸发除去乙醚后, 在相同条件 下进行反应, 所流失的Pd几乎没有催化作用.

总之,我们采用自然、可再生、无毒和对环境友好 的乳酸阴离子离子液体制备了PdNPs@[Bmim]Lac催化 剂, 方法简单. Pd纳米粒子高度分散在 $[\mathrm{Bmim}] \mathrm{Lac}$ 中, 平 均粒径为 2.2-3.1 nm. 催化剂可高效催化一系列芳基溴 化物和碘化物与不同烯烃的Heck-Mizoroki反应, 且可循 环使用6次. 